\title{
Will aspirin rescue us from cancer?
}

\author{
Ludovica Tagliabue $\cdot$ Mariateresa Pugliano
}

Received: 24 May 2011/ Accepted: 19 June 2011/Published online: 8 July 2011

(C) SIMI 2011

\section{Background}

In the developed world, the lifetime risk of cancer is about $40 \%$ [1] but, by contrast with treatment of cancer, there has been little progress in the use of drugs for prevention. Some grades of evidence suggest an association between a longterm use of aspirin and a reduction in the risk of some cancers, in particular gastrointestinal tumours [2-7]. However, as most of them derive from observational studies, it is important to note that these type of data have often been shown to be unreliable [8].

\section{Summary}

Rothwell and colleagues [9] studied the effect of aspirin on risk of fatal cancer and on overall all-cause mortality, using data extracted from other randomised trials that had a mean or median scheduled trial treatment of aspirin versus control of at least 4 years and a range extending beyond 5 years. Searching in Pubmed, Embase and Cochrane Library and using the data from the ATT collaboration search of 2002 [10], they found eight randomised clinical

On behalf of GrAM.

L. Tagliabue $(\bowtie)$

Dipartimento di Sanità Pubblica, Microbiologia e Virologia, Università di Milano,

Milan, Italy

e-mail: ludovica.tagliabue@gmail.com

\section{Pugliano}

Immunoematologia Trasfusionale, Dipartimento di Medicina Trasfusionale, Ospedale S. Paolo,

Milan, Italy trials (published between 1998 and 2010) that assessed the efficacy of aspirin (any dose) given for at least 4 years versus no aspirin, in the absence or in the presence of another antiplatelet or antithrombotic drug for the primary or secondary prevention of cardiovascular events. They contacted the original investigators to determine whether the data about the number of deaths for cancer as the main cause, the time from randomisation to death and the primary site of cancer were obtainable. If available, they used these individual patient data and assessed, with KaplanMeier curves and Cox proportional hazards model, the effect of aspirin on death due to cancer, stratified for the site of cancer (cancers of the gastrointestinal tract versus other solid cancers versus haematological cancers), for the length of the follow-up (more or less than 5 years from randomisation) and for the type of solid cancer (site and histological type). They also estimated pooled effect of aspirin on risk of cancer death and all-cause mortality using fixed-effects meta-analysis. All analyses were made by intention-to-treat.

The analysis of individual patient data, available from seven of the eight included trials (23,535 patients, 657 cancer deaths), reveals that aspirin significantly reduces deaths due to cancer (HR 0.82, 95\% CI 0.70-0.95, $p=0.01$ ), the benefit appears just after 5 years' follow-up (HR $0.66,95 \%$ CI $0.50-0.87, p=0.003$ ). The analysis of pooled data from all the eight included trials (counting 25,570 patients and 674 cancer deaths) reveals that allocation to aspirin reduces death due to cancer [pooled odds ratio (OR) $0.79,95 \%$ CI $0.68-0.92, p=0.003$ ], but the drug does not significantly decrease all-cause mortality (OR $0.92,95 \%$ CI $0.85-1.00, p=0.047$ ). Moreover, Rothwell and colleagues present data obtained from three trials performed in UK (12,659 patients; 1,634 deaths) with a follow-up of 20 years: the risk of cancer death remains 
lower in the aspirin groups than in the control groups (HR $0.80,95 \%$ CI $0.72-0.88, p<0.0001)$, and the benefits increase with scheduled duration of trial treatment ( $\geq 7.5$ years $0.69,95 \%$ CI $0.54-0.88, p=0.003$ ). The effect is unrelated to aspirin dose (75 $\mathrm{mg}$ upwards), sex or smoking, but increases with age at randomisation.

\section{Strengths of the study}

- The study handles the prevention of cancer using a widely used drug and evaluating it with hard and clinically important outcomes (death for cancer, overall mortality and time to death).

- In the 20 years follow-up analysis, the researchers review all the recorded causes of death of the patients with known incident cancer, in order to exclude any possible bias derived from an increased risk of vascular events due to withdrawal of aspirin treatment after a diagnosis of cancer, which could reduce the number of deaths attributed to cancer in the aspirin groups.

- Using data from the TPT trial [11], which compared the efficacy of aspirin versus placebo with that of warfarin versus placebo, the study excludes the influence of early diagnosis of cancer due to bleeding on the risk of death for adenocarcinoma during long-term follow-up. In the group treated with warfarin no effect is demonstrated, excluding that an early diagnosis could be a major source of bias.

\section{Weakness of the study}

- Rothwell and colleagues do not report any data on bleeding events: also if they excluded any possible bias derived from the influence of early diagnoses on cancer death, this adverse effect surely influences any clinical or public health choice, as aspirin is known to increase bleeding events [11], and this would be more probable after a treatment of 5 years. As reported by Moayyedi et al. [12], the authors quote a $20 \%$ reduction in death from cancer as a relative risk reduction (OR 0.79, 95\% CI 0.68-0.92), but after more than 5 years treatment the absolute reduction in death from cancer is only $0.5 \%$ with $\mathrm{NNT}=200$ (95\% CI 111-1,075). Without any data on bleeding, but a $50 \%$ increase in risk of serious bleeding described in primary prevention trials [11], the risk/benefit and the cost/effectiveness ratio are not clear.

- All the included original studies are randomised trials that evaluated the efficacy of daily aspirin for the primary and secondary prevention of vascular events, not designed for properly assessing the risk of death for cancer or cancer prevention.

\section{Question marks}

- Eligibility criteria for the inclusion of studies seem fairly arbitrary: neither the list of studies excluded nor the reason of their exclusion is reported. For example, it is not clear why the 10-year follow-up Women's Health Study [7], a randomised trial of $100 \mathrm{mg}$ of aspirin on alternate days versus control, and the Physicians' Health Study [13], a randomised trial of low-dose aspirin on alternate days on cardiovascular mortality, were not included. This information could be important for clarifying any selection bias.

- The assessment of risk factors for cancer of subjects included is not reported, probably lacking in the original papers. So, we cannot verify that risk factors for cancer are equally distributed in the two groups.

- We think that the reduction of all cause mortality in aspirin patients is a clinically important outcome, but it is probable that the weak significant result in this group is driven by the effect of the drug on reduction of cardiovascular deaths, as all patients in all the included trials were at high cardiovascular risk.

\section{Sponsorship}

- The study was unfunded and independent.

\section{Clinical bottom line}

Previous works reported a possible role of aspirin in cancer prevention, and this study provides further data in this direction. Nevertheless, the authors don't give enough information for making with certainty any clinical or public health choice, and the results are not robust enough to recommend aspirin for the general population. There is still little evidence about the effect of aspirin on cancer prevention, and, in particular, there are not enough data for properly evaluating the benefits against the risks. Finally, on the base of the data reported, we wonder if the authors are already taking daily aspirin.

Conflict of interest None.

\section{References}

1. Jemal A, Center MM, DeSantis C, Ward EM (2010) Global patterns of cancer incidence and mortality rates and trends. Cancer Epidemiol Biomarkers Prev 19:1893-1907 
2. Cuzick J, Otto F, Baron JA et al (2009) Aspirin and non-steroidal anti-inflammatory drugs for cancer prevention: an international consensus statement. Lancet Oncol 10:501-507

3. Bosetti C, Gallus S, La Vecchia C (2009) Aspirin and cancer risk: a summary review to 2007. Recent Results Cancer Res 181:231-251

4. Rothwell PM, Wilson M, Elwin CE, Norrving B et al (2010) Long-term effect of aspirin on colorectal cancer incidence and mortality: 20-year follow-up of five randomised trials. Lancet 376:1741-1750

5. Cole BF, Logan RF, Halabi S et al (2009) Aspirin for chemoprevention of colorectal adenomas: meta-analysis of the randomised trials. J Natl Cancer Inst 101:256-266

6. Chan AT, Ogino S, Fuchs CS (2007) Aspirin and risk of colorectal cancer in relation to expression of COX-2. N Engl J Med 356:2131-2142

7. Cook NR, Lee IM, Gaziano JM et al (2005) Low-dose aspirin in the primary prevention of cancer: the Women's Health Study: a randomised controlled trial. JAMA 294:47-55

8. Lawlor DA, Smith GD, Bruckdorfer KR, Kundu D et al (2004) Those confounded vitamins: what can we learn from the differences between observational versus randomised trial evidence? Lancet 363:1724-1727

9. Rothwell PM, Fowkes FG, Belch JF, Ogawa H, Warlow CP, Meade TW (2011) Effect of daily aspirin on long-term risk of death due to cancer: analysis of individual patient data from randomised trials. Lancet 2011:31-41

10. Antithrombotic Trialists' (ATT) Collaboration (2002) Collaborative meta-analysis of randomised trials of antiplatelet therapy for prevention of death, myocardial infarction, and stroke in high risk patients. BMJ 324:71-86

11. The Medical Research Council's General Practice Research Framework (1998) Thrombosis prevention trial: randomised trial of low-intensity oral anticoagulation with warfarin and low-dose aspirin in the primary prevention of ischaemic heart disease in men at increased risk. Lancet 351:233-241

12. Moayyedi P, Jankowski JA (2010) Does long term aspirin prevent cancer? BMJ 341:c7326

13. Steering Committee of the Physicians' Health Study Research Group (1989) Final report on the aspirin component of the ongoing Physicians' Health Study. N Engl J Med 321(3):129-135 